\title{
Various bifurcations in the development of stem cells
}

\author{
Lianyu Chen ${ }^{1, a}$, Ibrahim Ismael Hamarash ${ }^{2,3, b}$, Sajad Jafari ${ }^{4,5, c}$, Karthikeyan Rajagopal ${ }^{6, d}(\mathbb{D}$, and \\ Iqtadar Hussain ${ }^{7, e}$ \\ 1 School of Electrical and Information Engineering, Jiangsu University of Technology, Changzhou 213001, China \\ 2 Electrical Engineering Department, Salahaddin University-Erbil, Kirkuk Rd., Erbil, Kurdistan, Iraq \\ 3 School of Computer Science and Engineering, University of Kurdistan Hewler, 40m St., Erbil, Kurdistan, Iraq \\ 4 Biomedical Engineering Department, Amirkabir University of Technology, Tehran 15875-4413, Iran \\ ${ }^{5}$ Health Technology Research Institute, Amirkabir University of Technology, No.350, Hafez Ave, Valiasr Square, \\ Tehran 159163-4311, Iran \\ 6 Center for Nonlinear Systems, Chennai Institute of Technology, Chennai, India \\ 7 Department of Mathematics, Statistics and Physics, Qatar University, Doha 2713, Qatar
}

Received 2 January 2021 / Accepted 30 October 2021 / Published online 13 November 2021

(C) The Author(s), under exclusive licence to EDP Sciences, Springer-Verlag GmbH Germany, part of Springer Nature 2021

\begin{abstract}
Cell development from an undifferentiated stem cell to a differentiated one is essential in forming an organism. In this paper, various bifurcations of a stem cell during this process are studied using a model based on Furusawa and Kaneko's hypothesis. Furusawa and Kaneko's hypothesis tells that the gene expression of stem cells is chaotic. By developing to a differentiated cell, the gene expression in more order, which is the cause of losing pluripotency. In this model, the chaotic dynamics of gene expression in the stem cells become ordered during the developments. Various patterns and bifurcation points can be seen during development. The bifurcation points and their predictions during the process of cell development are studied in this paper. Some well-known critical slowing down indicators are used to show the variations of slowness during the cell's development and predict the bifurcation points. It is vital since the unexpected changes of the state can cause a disaster. All of the indicators have a proper trend by approaching the bifurcation points and faring away.
\end{abstract}

\section{Introduction}

Cells are the basic units that generate every organ. Without knowing the cells, the study of life is pointless. Stem cells are capable of developing any particular cell with their tasks. In other words, they are undifferentiated cells that can develop into any differentiated one [1]. This property makes them exceptional choices in medical applications [2]. Pluripotent stem cells go to an undirected path to become any differentiated cell. Furusawa and Kaneko have proposed a hypothesis on the development of stem cells. This hypothesis tells that the gene expression of stem cells is chaotic while developing to a differentiated cell, the gene expression in more order, which is the cause of losing pluripotency [3].

Modeling various phenomena in real life can help researchers to know more about their behaviors. Neuron models are one of the examples [4]. A photosensitive

\footnotetext{
${ }^{\mathrm{a}} \mathrm{e}-\mathrm{mail}$ : chenly4254@sina.com

b e-mail: ibrahim.hamarash@gmail.com

${ }^{\mathrm{c}}$ e-mail: sajadjafari83@gmail.com

d e-mail: karthikeyan.rajagopal@citchennai.net (corresponding author)

e e-mail: iqtadarqau@gmail.com
}

neuron was presented in [5]. The modeling of neurons under magnetic flow is an exciting topic [6]. Recently some researchers try to implement neuron models using electronic elements [7]. The application of a memristive neuron model under electromagnetic radiation was discussed in [8]. A chaotic cancer model was studied in [9]. Recently a chaotic map was proposed to model the cells' development [10]. Various bifurcations of the COVID-19 model were discussed in [11]. The dynamics of COVID-19 spreading were studied in [12].

Many chaotic systems can be implemented by electronic elements [13-15]. Implementation of a memristive system was studied in [16]. Chaotic systems can be used in encryption goals [17]. A random number generator based on chaotic dynamics was studied in [18]. Fluctuations of biological and physical data can be interpreted as the oscillations of chaotic dynamics [19-21]. So studying the dynamical properties of biological models are very important. Multistability is important in the investigation of dynamical systems [22-24]. Various multistability of a circuit was studied in $[25,26]$. Dynamical systems can show various dynamics $[27,28]$. Various dynamical properties of a chaotic system were discussed in [29]. Coexisting attractors of chaotic maps were studied in $[30,31]$. 
The bifurcation diagram is one of the most potent tools in nonlinear dynamics [32]. It reveals various attractors of the system as well as the route of dynamic variations. Also, bifurcation points are crucial. The variation of a system from one dynamic to another can be horrible if the environment is not preparing. Recently various methods for the prediction of bifurcation points are studied [33,34]. One of these methods is the Lyapunov exponent, which approaches zero by getting close to bifurcation points [35]. Estimation of Lyapunov exponents in a particular class of systems was discussed in [36]. Some of the most common indicators of bifurcation points are autocorrelation and kurtosis. However, they should be modified to predict bifurcations in a period-doubling route to chaos [37].

In this paper, we discuss different dynamics of the model of the development of cells and predict its various bifurcation points. The model is based on Furusawa and Kaneko's hypothesis. The model shows chaotic dynamics of gene expression changes to order dynamics in the process of development. Prediction of the system's bifurcations using three slowing down indicators is discussed.

\section{Studied model}

Evolving stem cells from an undifferentiated cell to a differentiated one with particular tasks have a complicated route, modeled as Eq. (1) [10]. This model was based on the hypothesis of Furuxawa and Kaneko [3]. It tells that the cell's state in its evolution by passing the time can have various dynamics. In a cell division, the cells' numbers are increased. However, the interaction between cells becomes more powerful. Furthermore, the cells go through simpler dynamics in their evolution.

$$
x_{k+1}=x_{k}+r \sin \left(x_{k}\right)
$$

This model is studied in $r \in[0,5.3]$. The system's different behaviors by parameter $r$ were studied, such as equilibria, periodic, chaotic, and biotic dynamics. This model has been discussed in various studies [38]. Figure 1 shows the bifurcation diagram of Eq. (1) with $r$. This parameter is the feature of pluripotency.

The model shows various dynamics by decreasing parameter $r$ in the evolution of cells. In large parameters, the cells have pluripotent dynamics in a wide interval, which is called bios. Decreasing parameter makes the cells divided into various differentiated chaotic dynamics. Again decreasing parameter $r$ causes simple dynamics such as periodic and equilibrium point, which is the most differentiated version of cell dynamics. In this state, each cell acts its particular tasks and can not change to another one. When the cell is in a pluripotent dynamic (bios), it can transform into any unique dynamics (various chaotic and per attractors). However, after the transition, the system becomes more special until it receives the equilibrium points. In other words, the cell dynamics vary from a biotic one to

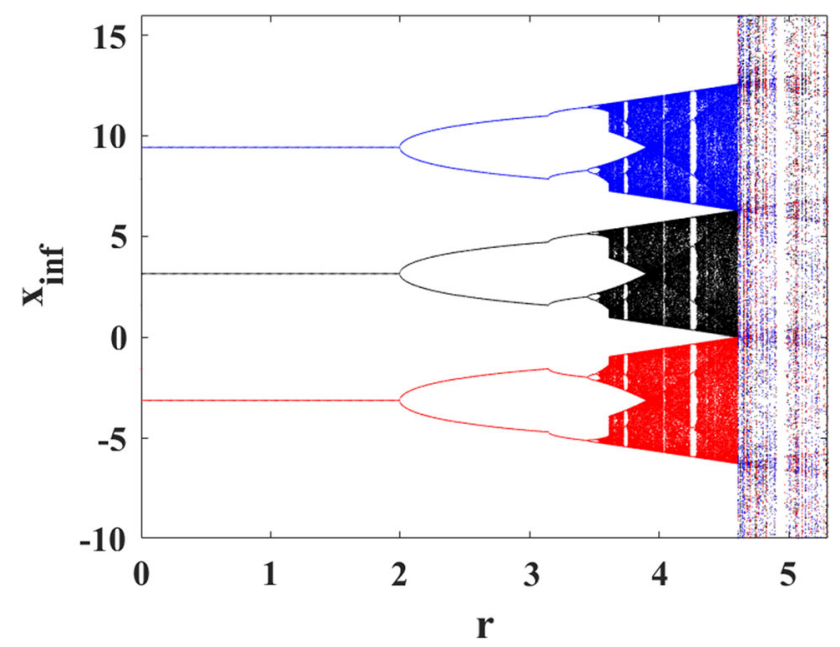

Fig. 1 Bifurcation diagram of Eq. (1) by changing $r$ and three initial values as $x_{0}=\frac{\pi}{2}$ for black dynamics, $x_{0}=\frac{5 \pi}{2}$ for blue dynamics, and $x_{0}=-\frac{\pi}{2}$ for red ones

chaotic ones and then to order dynamics by changing the feature of pluripotency. The model has many coexisting dynamics, and three of them are shown in Fig. 1 using three initial conditions.

\section{Indicating bifurcation points of the cell}

The development of cells has various bifurcation points like many other biological systems. The occurrence of bifurcation points can be a disaster if we do not have any information about them. It leads researchers to study the prediction of bifurcation points. In the previous section, various dynamics of the cell model were discussed . Here, the bifurcations of the Eq. (1) and the prediction of these variations are discussed. Three methods are used to study the bifurcation points of the studied model. Each method is applied to the system's three various studied routes from pluripotent stem cells to differentiated ones. However, we know that many other routes have the same properties as the three studied ones. In the rest of the paper, the left y-label presents the values of $x$ variable, and the right y-label presents the studied indicators' values. Also, all methods are applied to each of the three attractors' data, as discussed in Fig. 1.

The first method is the modified autocorrelation (AC) with lag-1. Autocorrelation of signal $S$ at lag-1 is defined as $A C=\frac{\mathrm{E}\left[\left(S_{t}-\mu\right)\left(S_{t+1}-\mu\right)\right]}{\sigma^{2}}$, where $\mu, \sigma^{2}$ are mean and variance of the signal. AC measures the memory of dynamics. In the modified method, the signal's period is estimated, and the autocorrelation is applied to the subvectors of various cycles. Figure 2 shows the bifurcation diagram (left y-label) and autocorrelation (right ylabel) of the black attractors. By approaching the bifurcation points, the $\mathrm{AC}$ approaches one. So it warns the prediction of bifurcation points before its occurrence. 
Fig. 2 Bifurcation diagram of Eq. (1) by changing $r$ and $x_{0}=\frac{\pi}{2}$ (left y-label). The green measure is the absolute value of the modified $\mathrm{AC}$ measure (right y-label)

Fig. 3 Bifurcation diagram of Eq. (1) by changing $r$ and $x_{0}=-\frac{\pi}{2}$ (left y-label). The green measure is the absolute value of the modified $\mathrm{AC}$ measure (right y-label)

Fig. 4 Bifurcation diagram of Eq. (1) by changing $r$ and $x_{0}=\frac{5 \pi}{2}$ (left y-label). The green measure is the absolute value of the modified $\mathrm{AC}$ measure (right y-label)
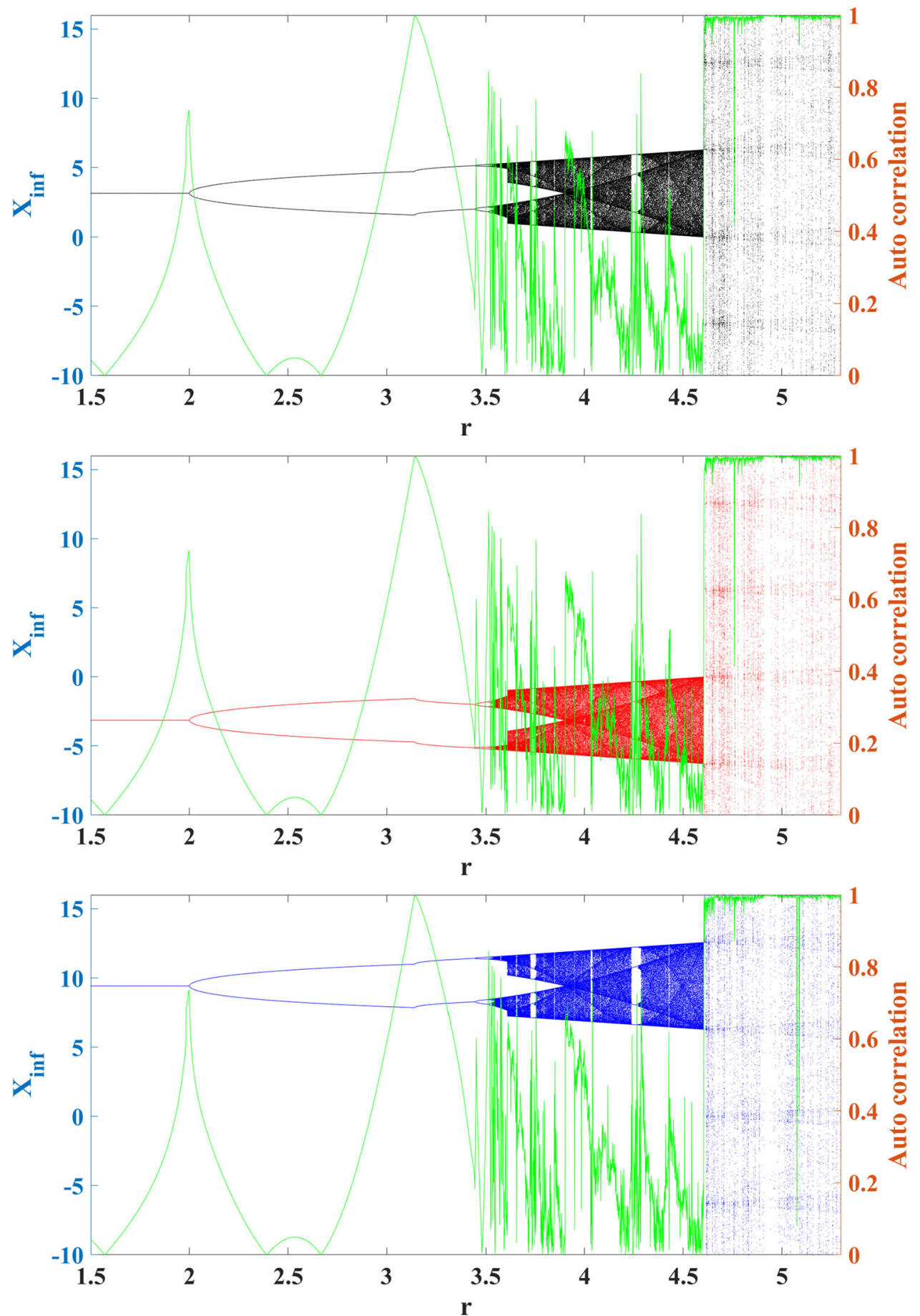

Various bifurcations are predicted. However, it cannot predict bifurcation from biotic dynamics to chaotic one.

The measure is calculated for the data of the three studied attractors. Figure 3 shows AC calculated for the red route and Fig. 4 for the blue one. These measures show reliable predictions in the bifurcation points of the period-doubling route to chaos, but not a meaningful trend for bios' transition to chaos.

The other measure used in the prediction of bifurcations of the cell model is kurtosis $(K) . K$ is the fourth moment as $k=\frac{\frac{1}{n} \sum_{t=1}^{n}\left(S_{t}-\mu\right)^{3}}{\sqrt{\frac{1}{n} \sum_{t=1}^{n}\left(S_{t}-\mu\right)^{2}}}$. It is based on the variations of the tail of data distribution. It decreased when approaching a bifurcation point. Here, the modified kurtosis is used, calculated on the vectors of extracted cycles, as discussed in the AC method. Figure 5 shows the kurtosis method, which is calculated for the data of black attractors. By approaching the bifurcation points, $\mathrm{K}$ decreases, and by getting away from them, it increases.

Figures 6 and 7 show the $K$ measure for red and blue attractors. The measure has the same trend in approaching and getting away from bifurcation points discussed in the black attractor. However, in the transi- 
Fig. 5 Bifurcation diagram of Eq. (1) by changing $r$ and $x_{0}=\frac{\pi}{2}$ (left y-label). The green measure is the logarithm of the modified $\mathrm{K}$ measure (right y-label)
Fig. 6 Bifurcation diagram of Eq. (1) by changing $r$ and $x_{0}=-\frac{\pi}{2}$ (left y-label). The green measure is the logarithm of the modified $\mathrm{K}$ measure (right y-label)
Fig. 7 Bifurcation diagram of Eq. (1) by changing $r$ and $x_{0}=\frac{5 \pi}{2}$ (left y-label). The green measure is the logarithm of the modified $\mathrm{K}$ measure (right y-label)
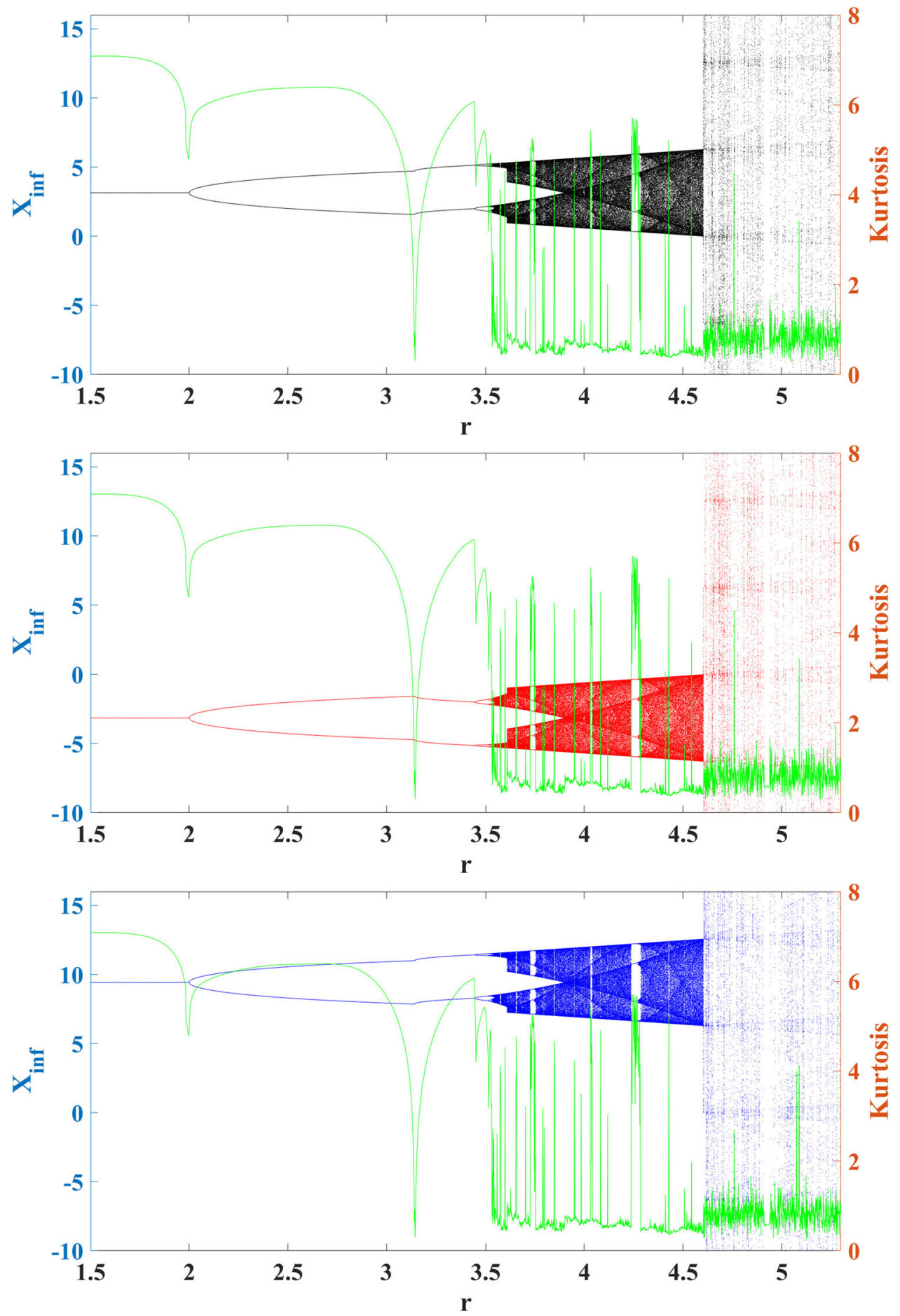

tion from biotic dynamics to chaotic ones, the measure does not show any notable trend.

The last measure which is used here is the Lyapunov exponent (LY). It was recently presented as an indicator of bifurcation points. However, its calculations have some issues. Lyapunov exponent applied to the raw time series and can predict various bifurcation points. Its value is zero in the bifurcation points.

Figure 8 presents the LY of the cell model calculated for the data of the black attractor. The LY measure approaches zero in various bifurcation points. So LY can predict various bifurcations, but it fails to predict bifurcation from bios to chaos. Figures 9 and 10 show the LY measure calculated for the red and blue attractors. They show the same pattern in the prediction of bifurcations.

An impressive result was that the values of indicators for each of the three studied dynamics (black, red, and blue) were the same, which means that they all have the same dynamical properties. However, they approach various differentiated cells with various tasks. 
Fig. 8 Bifurcation diagram of Eq. (1) by changing $r$ and $x_{0}=\frac{\pi}{2}$ (left y-label). The green measure is the LY measure (right y-label)

Fig. 9 Bifurcation diagram of Eq. (1) by changing $r$ and $x_{0}=-\frac{\pi}{2}$ (left y-label). The green measure is the LY measure (right y-label)

Fig. 10 Bifurcation diagram of Eq. (1) by changing $r$ and $x_{0}=\frac{5 \pi}{2}$ (left y label). The green measure is the LY measure (right y label)
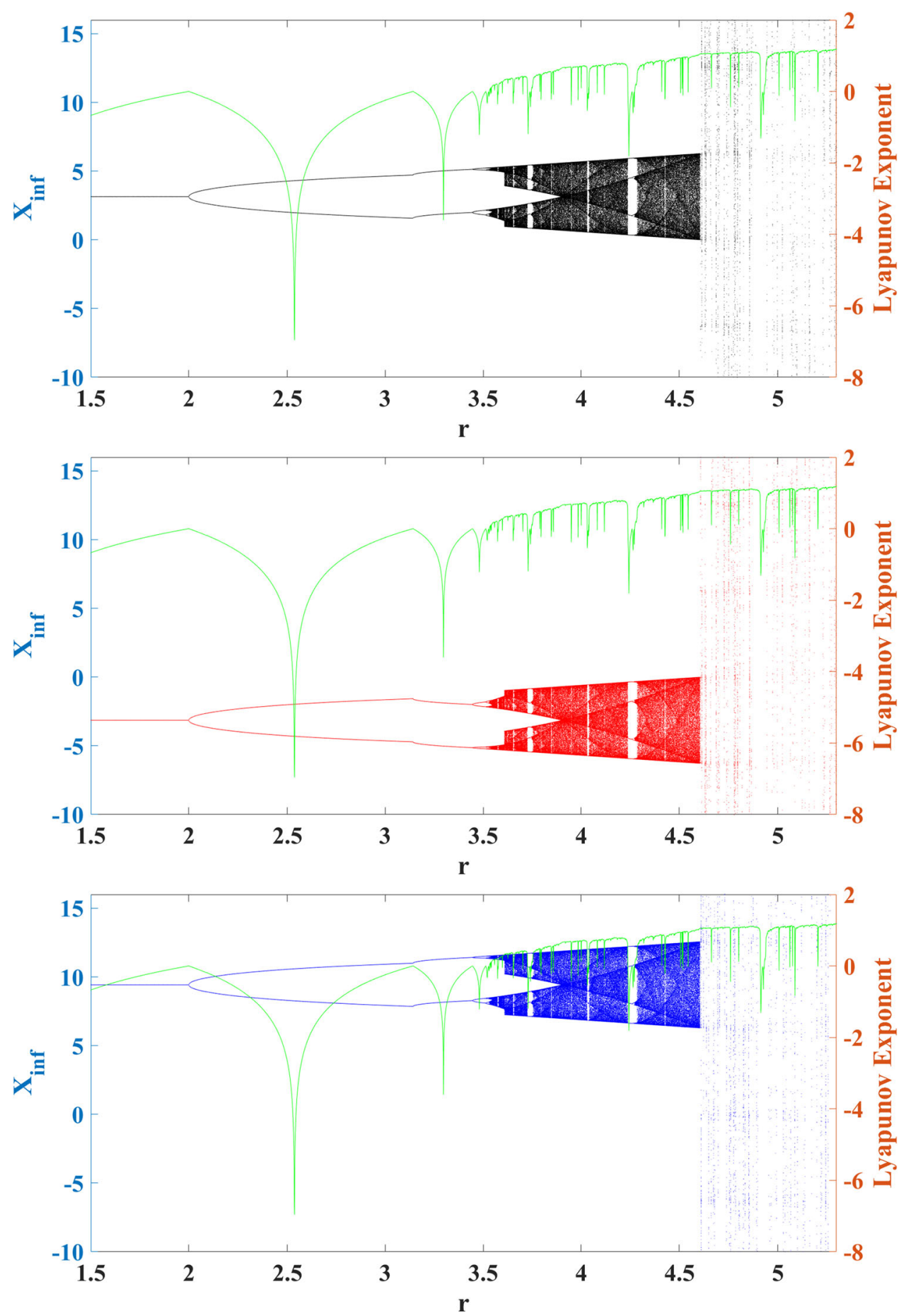

Comparing these three indicators show that all of them have a proper trend by approaching the bifurcation points and faring away from them. In theory, AC should be one in the bifurcation points, and $\mathrm{K}$ should be zero. However, in numerical calculations, AC and $\mathrm{K}$ do not satisfy these values in some of the bifurcation points. Lyapunov exponent approaches zero, and it can be seen in the bifurcation points.

\section{Conclusion}

Here various bifurcation points of a model for the cell dynamics were studied. The system has presented the behavior of stem cells' time evolution from pluripotent cells to differentiated ones with a particular task. The model was based on Furusawa and Kaneko's hypothesis. It has shown that the biotic dynamics of stem cells' gene expression became chaotic, periodic, and equilibrium points in the time evolution. So the system had various bifurcation points. Three indicators were used to 
predict these points: modified autocorrelation, modified kurtosis, and Lyapunov exponent. Their results have shown that these measures can predict different bifurcations in the period-doubling route to chaos. However, they did not have any trend in the bifurcation of biotic dynamics to chaotic ones. The results have also presented that the indicators had the same pattern in predicting bifurcations of the three studied dynamics (black, red, and blue). Comparing these three indicators has shown that all of them have a proper trend by approaching the bifurcation points and faring away from them in the period-doubling route to chaos. In theory, autocorrelation should be one in the bifurcation points, and kurtosis should be zero. However, in numerical calculations, $\mathrm{AC}$ and $\mathrm{K}$ did not satisfy these values in some of the bifurcation points. Lyapunov exponent approaches zero, and it can be seen in the bifurcation points.

Acknowledgements This work is funded by the Center for Nonlinear Systems, Chennai Institute of Technology, India, vide funding number CIT/CNS/2021/RP-017.

\section{References}

1. V.K. Gangaraju, H. Lin, MicroRNAs: key regulators of stem cells. Nat. Rev. Mol. Cell Biol. 10, 116-125 (2009)

2. N. Rajabzadeh, E. Fathi, R. Farahzadi, Stem cell-based regenerative medicine. Stem. Cell Investig.vol. 6 (2019)

3. C. Furusawa, K. Kaneko, Chaotic expression dynamics implies pluripotency: when theory and experiment meet. Biol. Direct 4, 1-11 (2009)

4. J. Ma, J. Tang, A review for dynamics of collective behaviors of network of neurons. Sci. Chin. Technol. Sci. 58, 2038-2045 (2015)

5. Y. Liu, W. -j. Xu, J. Ma, F. Alzahrani, A. Hobiny, A new photosensitive neuron model and its dynamics. Front. Inf. Technol. Electron. Eng. pp. 1-10 (2020)

6. M. Lv, C. Wang, G. Ren, J. Ma, X. Song, Model of electrical activity in a neuron under magnetic flow effect. Nonlinear Dyn. pp. 1-12 (2016)

7. X. Hu, C. Liu, L. Liu, J. Ni, S. Li, An electronic implementation for Morris-Lecar neuron model. Nonlinear Dyn. 84, 2317-2332 (2016)

8. S. Zhang, J. Zheng, X. Wang, Z. Zeng, Multi-scroll hidden attractor in memristive $\mathrm{HR}$ neuron model under electromagnetic radiation and its applications. Chaos Interdiscip. J. Nonlinear Sci. 31, 011101 (2021)

9. S. Khajanchi, M. Perc, D. Ghosh, The influence of time delay in a chaotic cancer model. Chaos Interdiscip. J. Nonlinear Sci. 28, 103101 (2018)

10. F. Nazarimehr, S.S. Hosseini, A.J.M. Khalaf, S. Jafari, J.C. Sprott, Process equation as a model for the development of cells. Eur. Phys. J. Spec. Top. 229, 921-927 (2020)

11. F. Nazarimehr, V.-T. Pham, T. Kapitaniak, Prediction of bifurcations by varying critical parameters of COVID19. Nonlinear Dyn. pp. 1-12 (2020)

12. S. Boccaletti, W. Ditto, G. Mindlin, A. Atangana, Modeling and forecasting of epidemic spreading: the case of
Covid-19 and beyond. Chaos Solit. Fract. 135, 109794 (2020)

13. B. Ramakrishnan, A. Durdu, K. Rajagopal, A. Akgul, Infinite attractors in a chaotic circuit with exponential memristor and Josephson junction resonator. AEU-Int. J. Electron. Commun. 123, 153319 (2020)

14. Z. Wei, I. Moroz, J. Sprott, A. Akgul, W. Zhang, Hidden hyperchaos and electronic circuit application in a 5D self-exciting homopolar disc dynamo. Chaos: Interdiscip. J. Nonlinear Sci. 27, 033101 (2017)

15. G. Leutcho, J. Kengne, L.K. Kengne, Dynamical analysis of a novel autonomous 4-D hyperjerk circuit with hyperbolic sine nonlinearity: chaos, antimonotonicity and a plethora of coexisting attractors. Chaos Solit. Fract. 107, 67-87 (2018)

16. Q. Lai, Z. Wan, A. Akgul, O.F. Boyraz, M.Z. Yildiz, Design and implementation of a new memristive chaotic system with application in touchless fingerprint encryption. Chin. J. Phys. 67, 615-630 (2020)

17. M.Z. Yildiz, O. Boyraz, E. Guleryuz, A. Akgul, I. Hussain, A novel encryption method for dorsal hand vein images on a microcomputer. IEEE Access 7, 6085060867 (2019)

18. L. Moysis, A. Tutueva, K. Christos, D. Butusov, A chaos based pseudo-random bit generator using multiple digits comparison. Chaos Theory Appl. 2, 58-68 (2020)

19. W. Znegui, H. Gritli, S. Belghith, Design of an explicit expression of the Poincaré map for the passive dynamic walking of the compass-gait biped model. Chaos Solit. Fract. 130, 109436 (2020)

20. H. Gritli, N. Khraief, S. Belghith, Chaos control in passive walking dynamics of a compass-gait model. Commun. Nonlinear Sci. Numer. Simul. 18, 2048-2065 (2013)

21. D. Costa, V. Vaziri, M. Kapitaniak, S. Kovacs, E. Pavlovskaia, M. A. Savi, et al., Chaos in impact oscillators not in vain: Dynamics of new mass excited oscillator. Nonlinear Dyn. (2020)

22. H. Bao, M. Chen, H. Wu, B. Bao, Memristor initialboosted coexisting plane bifurcations and its extreme multi-stability reconstitution in two-memristor-based dynamical system. Sci. Chin. Technol. Sci. pp. 1-11 (2019)

23. H. Bao, N. Wang, B. Bao, M. Chen, P. Jin, G. Wang, Initial condition-dependent dynamics and transient period in memristor-based hypogenetic jerk system with four line equilibria. Commun. Nonlinear Sci. Numer. Simul. 57, 264-275 (2018)

24. Z. NJITACKE, T. FOZIN, L. K. KENGNE , G. LEUTCHO, E. M. Kengne, J. Kengne, "Multistability and its Annihilation in the Chua's Oscillator with Piecewise-Linear Nonlinearity," Chaos Theory and Applications, vol. 2, pp. 77-89, 2020

25. B.-C. Bao, Q. Xu, H. Bao, M. Chen, Extreme multistability in a memristive circuit. Electron. Lett. 52, 10081010 (2016)

26. M. Chen, M. Sun, H. Bao, Y. Hu, B. Bao, Flux-charge analysis of two-memristor-based Chua's circuit: dimensionality decreasing model for detecting extreme multistability. IEEE Trans. Ind. Electron. (2019)

27. J. Cantisán, J. M. Seoane, M. A . Sanjuán, Transient dynamics of the Lorenz system with a parameter drift. arXiv preprint arXiv:2009.11017 (2020) 
28. R. Capeáns, J. Sabuco, M.A. Sanjuán, J.A. Yorke, Partially controlling transient chaos in the Lorenz equations. Philos. Trans. R. Soc. A: Math. Phys. Eng. Sci. 375, 20160211 (2017)

29. Y. Adiyaman, S. Emroglu, M. K. UÇar, M. Yildiz, Dynamical analysis, electronic circuit design and control application of a different chaotic system. Chaos Theory Appl. 2, 8-14

30. H. Bao, Z. Hua, N. Wang, L. Zhu, M. Chen, B. Bao, Initials-boosted coexisting Chaos in a 2-D sine map and its hardware implementation. IEEE Trans. Ind. Inf. 17, 1132-1140 (2021)

31. H. Li, Z. Hua, H. Bao, L. Zhu, M. Chen, B. Bao, Twodimensional memristive hyperchaotic maps and application in secure communication. IEEE Trans. Ind. Electron. pp. 1-1 (2020)

32. J. C. Sprott, J. C. Sprott, Chaos and time-series analysis vol. 69: Citeseer (2003)

33. M. Scheffer, J. Bascompte, W.A. Brock, V. Brovkin, S.R. Carpenter, V. Dakos et al., Early-warning signals for critical transitions. Nature 461, 53 (2009)

34. V. Dakos, Nature's dynamical complexity. Nat. Ecol. Evolut. 4, 12-13 (2020)
35. F. Nazarimehr, A. Ghaffari, S. Jafari, S.M.R.H. Golpayegani, Sparse recovery and dictionary learning to identify the nonlinear dynamical systems: one step toward finding bifurcation points in real systems. Int. J. Bifurc. Chaos 29, 1950030 (2019)

36. D.M.B. Netto, A. Brandão, A. Paiva, P.M. Pacheco, M.A. Savi, Estimating Lyapunov spectrum on shapememory alloy oscillators considering cloned dynamics and tangent map methods. J. Br. Soc. Mech. Sci. Eng. 42, 1-13 (2020)

37. F. Nazarimehr, S. Jafari, S. M. R. Hashemi Golpayegani, M. Perc, J. C. Sprott ,Predicting tipping points of dynamical systems during a period-doubling route to chaos. Chaos Interdiscip. J. Nonlinear Sci. 28, 073102 (2018)

38. F. Nazarimehr, S. Jafari, S.M.R.H. Golpayegani, L.H. Kauffman, Investigation of Bifurcations in the process equation. Int. J. Bifurc. Chaos 27, 1750201 (2017) 